\title{
The role of IL-1 in the regulation of copeptin in patients with metabolic syndrome
}

\author{
Milica Popovic ${ }^{1,2}$, Fahim Ebrahimi ${ }^{1,2}$, Sandrine Andrea Urwyler ${ }^{1,2}$, Marc Yves Donath ${ }^{1,3}$ and Mirjam Christ-Crain ${ }^{1,2}$ \\ ${ }^{1}$ Department of Endocrinology, Diabetology and Metabolism, University Hospital Basel, Basel, Switzerland \\ ${ }^{2}$ Department of Clinical Research, University of Basel and University Hospital Basel, Basel, Switzerland \\ ${ }^{3}$ Department of Biomedicine, University of Basel, Basel, Switzerland
}

Correspondence should be addressed to M Christ-Crain: mirjam.christ-crain@usb.ch

\begin{abstract}
Arginine vasopressin (AVP) was suggested to contribute to cardiovascular risk and type 2 diabetes in patients with metabolic syndrome. The proinflammatory cytokine interleukin (IL)-1 is able to induce AVP secretion and plays a causal role in cardiovascular mortality and type 2 diabetes. We investigated in two studies whether copeptin levels - the surrogate marker for AVP - are regulated by IL-1-mediated chronic inflammation in patients with metabolic syndrome. Study A was a prospective, interventional, single-arm study (2014-2016). Study B was a randomized, placebo-controlled, double-blind study (2016-2017). $n=73$ (Study A) and $n=66$ (Study B) adult patients with metabolic syndrome were treated with $100 \mathrm{mg}$ anakinra or placebo (only in study B) twice daily for 1 day (study A) and 28 days (study B). Fasting blood samples were drawn at day 1, 7, and 28 of treatment for measurement of serum copeptin. Patients with chronic low-grade inflammation (C-reactive protein levels $\geq 2 \mathrm{mg} / \mathrm{L}$ ) and $\mathrm{BMI}>35 \mathrm{~kg} / \mathrm{m}^{2}$ had higher baseline copeptin levels (7.7 (IQR 4.9-11.9) vs 5.8 (IQR 3.9-9.3) pmol/L, $P_{\text {inflamm }}=0.009 ; 7.8$ (IQR 5.4-11.7) vs 4.9 (IQR 3.7-9.8) $\mathrm{pmol} / \mathrm{L}, P_{\mathrm{BMI}}=0.008$ ). Copeptin levels did not change either in the anakinra or in the placebo group and remained stable throughout the treatment $(P=0.44)$. Subgroup analyses did not reveal effect modifications. Therefore, we conclude that, although IL-1-mediated inflammation is associated with increased circulating copeptin levels, antagonizing IL-1 does not significantly alter copeptin levels in patients with metabolic syndrome.
\end{abstract}

\section{Key Words}

- metabolic syndrome

- copeptin

- arginine vasopressin

- interleukin-1

- low-grade inflammation

\section{Introduction}

Patients with metabolic syndrome are at significant risk for developing type 2 diabetes mellitus and cardiovascular diseases (1). Arginine vasopressin (AVP) was suggested to play a causal role in the development of type 2 diabetes mellitus and cardiovascular disease (2). Indeed, several studies have demonstrated that copeptin - the C-terminal part of the AVP precursor and surrogate marker for AVP (3) - predicts insulin resistance and onset of type 2 diabetes mellitus $(4,5,6,7,8,9,10)$ and is associated with an increased cardiovascular mortality in patients with metabolic syndrome $(5,11,12,13)$. There are several potential pathways by which AVP might mediate cardiovascular risk and type 2 diabetes. First, AVP leads to an amplification of cortisol release by inhibiting negative feedback on adrenocorticotropic hormone secretion from the anterior pituitary gland and by direct stimulation of the adrenal cortex $(14,15)$. Moreover, AVP induces epinephrine secretion by stimulation of the chromaffin cells in the adrenal medulla (16) and stimulates glycogenolysis and gluconeogenesis via V1a receptors in the liver (17). Furthermore, AVP has antilipolytic (18) and prothrombotic (19) effects and mediates coronary vasoconstriction through vasopressin 1 a receptors (20).

The mechanisms underlying the upregulation of AVP/ copeptin levels in patients with metabolic syndrome are unknown. Interestingly, copeptin seems to be strongly

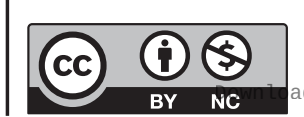


associated with elevated levels of C-reactive protein (CRP) $(8,21,22,23)$. Elevated CRP levels in patients with metabolic syndrome result from a chronic activation of the IL-1 system, a key cytokine stimulated by metabolic stress (24). Chronic low-grade inflammation enacted through IL-1 activity was recently shown to play a causal role in the development of both type 2 diabetes mellitus and cardiovascular disease. Randomized treatment with IL-1 antagonists has proven capacity to reduce HbA1c levels in patients with type 2 diabetes mellitus and to reduce cardiovascular mortality in patients with coronary heart disease and elevated levels of CRP $(25,26)$. Interestingly, there seems to be an interplay between IL-1 and AVP, since animal experiments have shown induction of AVP secretion in response to IL-1 application $(27,28$, 29 ), although there is conflicting data in a rat model of sepsis (30).

We, therefore, hypothesize that the increased levels of copeptin observed in patients with metabolic syndrome are caused by an overactivity of IL-1 and that antagonism of the IL-1 pathway would lead to a reduction of copeptin levels. In this study, we report the results of two interventional trials investigating effects of IL-1 antagonism in obese individuals with metabolic syndrome.

\section{Methods}

This is a preplanned secondary analysis of two interventional trials $(31,32)$. Both trials were conducted according to the ethical guidelines of the Declaration of Helsinki and the applicable International Conference on Harmonization (ICH) guidelines on good clinical practice. Both trials were approved by the Ethics Committee Northwest and Central Switzerland (EKNZ) and Swissmedic and were registered on ClinicalTrials.gov (NCT00757276, NCT02672592). All patients provided written informed consent. Patients were recruited at two tertiary care centers in Switzerland (University Hospital Basel and Kantonsspital Aarau).

\section{Study A: Patients and trial design}

Study A was a prospective, open-labeled, interventional trial investigating short-term ( $=1$ day) effects of IL-1 receptor antagonism in 73 obese patients with metabolic syndrome. Detailed study procedures have been published previously (32). Briefly, inclusion criteria were age between 18 and 80 years, body-mass index (BMI) $>30 \mathrm{~kg} / \mathrm{m}^{2}$ and at least one of the following additional features of the metabolic syndrome: Hyperglycemia (HbA1c $>5.7 \%$ ), hypertension (blood pressure (BP) $>130 / 85 \mathrm{mmHg}$ or $\mathrm{BP}$ lowering therapy), or dyslipidemia (HDL-C $<1.0 \mathrm{mmol} / \mathrm{L}$ or triglycerides $>1.7 \mathrm{mmol} / \mathrm{L}$ or low-density-lipoproteincholesterol (LDL-C) $>3.4 \mathrm{mmol} / \mathrm{L}$ or lipid lowering treatment). Main exclusion criteria were concurrent medication with glucocorticoids, known Cushing's syndrome, an underlying chronic inflammatory disease, history of a severe infection within the previous 2 months or a current infection, severe comorbidities and pregnancy or breastfeeding. After the screening visit, all patients received three s.c. injections of the recombinant human interleukin-1-receptor antagonist anakinra/Kineret ${ }^{\circledR} 100$ $\mathrm{mg}$ within 2 days. The consecutive injections were started at 20:00 $\mathrm{h}$ and continued in a time interval of $12 \mathrm{~h}$.

The study-visits assessed in this analysis were 'Baseline' (='Day 0') and after three injections of the IL-1 antagonist anakinra/Kineret ${ }^{\circledR}\left(='\right.$ Day $\left.1^{\prime}\right)$. Study visits were scheduled in the morning between 07:30 $\mathrm{h}$ and 10:00 $\mathrm{h}$ after an overnight fast and blood samples were drawn at both visits.

\section{Study B: Patients and trial design}

Study B was a randomized, placebo-controlled, doubleblind, interventional trial investigating short- as well as long-term effects of IL-1 receptor antagonism in 67 patients. A detailed description of study procedures was published previously (31). Main eligibility criteria were similar to study A. As study B was primarily designed to investigate the effect of IL-1 antagonism on testosterone levels in obese men with low levels of testosterone, all patients were male, aged 18 to 75 years with a BMI $>30$ $\mathrm{kg} / \mathrm{m}^{2}$ and total testosterone levels $<12 \mathrm{nmol} / \mathrm{L}$. Patients were randomized 1:1 to receive either anakinra/Kineret ${ }^{\circledR}$ $100 \mathrm{mg}$ or placebo as s.c. injection twice daily in a time interval of $12 \mathrm{~h}$. Study visits were scheduled in the morning at baseline (day 0), at day 1 (short-term visit), at day 7 and at 4 weeks. Blood samples were drawn at every visit. Therefore, patients were instructed not to eat or drink after midnight before the study visits in both studies.

\section{Laboratory analyses}

Routine laboratory parameters, that is, total cholesterol, HDL cholesterol, HbA1c, and triglyceride levels,

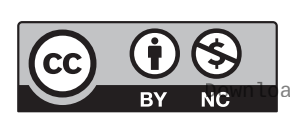

This work is licensed under a Creative Commons Attribution-NonCommercial 4.0 International License. ded from Bioscientifica.com at 04/26/2023 09:38:11AM via free access 
were analyzed at the local laboratories of the participating centers. At the University Hospital Basel, all routine parameters were measured on the Cobas 8000 c502 (Roche). At the Kantonsspital Aarau, total and HDL cholesterol were measured by immunoassay on the Architect i2000SR (Abbott), and HbA1c analysis was performed with the D-100 testing systems by BIORAD. In both centers, LDL cholesterol levels were calculated using the Friedewald formula (33).

CRP was determined with an immunoturbidimetric assay (Tina-quant C-Reactive Protein Gen. 3 Test; Roche Diagnostics $\mathrm{GmbH}$ ). Plasma copeptin levels were measured with a commercial automated immunofluorescence assay (B.R.A.H.M.S Copeptin-proAVP KRYPTOR, BRAHMS $\mathrm{GmbH}$, part of Thermo Fisher Scientific) in a batch analysis. For this, EDTA blood samples were centrifuged at $4{ }^{\circ} \mathrm{C}$ and stored at $-76{ }^{\circ} \mathrm{C}$.

\section{Statistical analysis}

Copeptin values were log-transformed in order to achieve a distribution analog to normal distribution. Thereby, one patient had to be excluded due to an extreme value at baseline of $129 \mathrm{pmol} / \mathrm{L}$, resulting in $n=66$ patients for Study B. Unless stated otherwise, categorical variables are expressed as count (percentage) and continuous variables as means ( \pm S.D.).

In a first analysis, we aimed to investigate which parameters are associated with higher copeptin levels at baseline. Therefore, linear regression models were calculated with log-transformed copeptin as dependent variable and the respective parameter as explanatory variable. All analyses were adjusted for sex. Afterwards, the statistically significant variables were selected for the combined analysis. These variables were combined (=added) as explanatory variables in one linear regression model to investigate which factors are independently associated with higher copeptin.

To assess treatment effects on copeptin levels, we used a linear mixed effects model with treatment group and baseline copeptin levels as fixed effect and participant ID as random effect. To investigate whether defined subgroups of patients responded differently to treatment with anakinra, we conducted subgroup analyses. For this, linear regression models were calculated with logtransformed copeptin as dependent variable and the following interaction term as explanatory variable: Treatment group * subgroup variable. These analyses were adjusted for baseline copeptin levels, treatment day, and sex. All $P$-values are two-sided and have not been adjusted for multiple testing.

Statistical analyses were performed and graphs drawn using R i386 Version 3.4.1.

\section{Results}

\section{Baseline characteristics}

Baseline characteristics of the 73 patients in study A and 66 patients in study B are shown in Table 1. In study B, the baseline characteristics were well-balanced between the two treatment groups. To summarize briefly, $49.3 \%$ vs $100 \%$ of the patients were male in Study A and B, respectively. Mean age was 54 years. Mean BMI was approximately $37 \mathrm{~kg} / \mathrm{m}^{2}$ with nearly all patients having visceral obesity. Around $70 \%$ of the patients presented with chronic low-grade inflammation (defined by a CRP level of $\geq 2 \mathrm{mg} / \mathrm{L}$ ). In study $\mathrm{A}, 86.3 \%$ of the patients suffered from either prediabetes or type 2 diabetes compared to $51.5 \%$ in study B. Furthermore, $72 \%$ of the patients in study A were treated with antihypertensive medication, whereas $45 \%$ had antihypertensive drugs in study B.

\section{Baseline copeptin levels}

Figure 1 shows baseline copeptin levels for different subgroups. Patients with chronic low-grade inflammation had significantly higher median copeptin levels than those without (7.7 (IQR 4.9-11.9) vs 5.8 (IQR 3.9-9.3) pmol/L, $P=0.009$, Fig. 1a). We observed an increase in median copeptin levels in dependence of diabetic status, that is, median copeptin levels for patients without diabetes were 6.0 (IQR 4.7-8.0) pmol/L, for those with prediabetes 7.3 (IQR 4.9-11.0) $\mathrm{pmol} / \mathrm{L}$, and 8.5 (IQR 4.0-14.1) pmol/L for patients with overt type 2 diabetes (overall $P=0.03$, Fig. 1b). The same held true for patients with and without (1) antihypertensive treatment (7.7 (IQR 4.8-13.1) vs 6.4 (IQR 4.2-9.1) pmol/L, $P=0.013$, Fig. 1c), (2) lipid-lowering treatment (8.8 (IQR 4.5-11.6) vs 6.6 (IQR 4.3-9.5) pmol/L, $P=0.009$, Fig. 1d), and (3) for patients with BMI above or below $35 \mathrm{~kg} / \mathrm{m}^{2}$ (7.8 (IQR 5.4-11.7) vs 4.9 (IQR 3.7-9.8) $\mathrm{pmol} / \mathrm{L}, P=0.008$, Fig. 1e). When adding all the investigated parameters (i.e. chronic low-grade inflammation, diabetic status, antihypertensive, and lipid-lowering treatment, and BMI categories) in one model, only chronic low-grade inflammation and BMI remained significantly associated with high copeptin $\left(P_{\text {Inflamm }}<0.01, P_{\mathrm{BMI}}=0.02\right)$.
This work is licensed under a Creative Commons Attribution-NonCommercial 4.0 International License. ded from Bioscientifica.com at 04/26/2023 09:38:11AM via free access 
Table 1 Baseline characteristics.

\begin{tabular}{l}
\hline Variable \\
\hline Treatment group \\
Age \\
Male sex \\
BMI (kg/m²) \\
Visceral obesity \\
Alcohol consumption (glasses/week) \\
Smokers \\
Ethnicity (Caucasian) \\
Systolic blood pressure (mmHg) \\
Diastolic blood pressure (mmHg) \\
Heart rate (bpm) \\
CRP (mg/L) \\
Chronic low-grade inflammation \\
Cholesterol, total (mmol/L) \\
HDL-C (mmol/L) \\
LDL-C (mmol/L) \\
Copeptin (pmol/L) \\
HbA1c (\%) \\
Triglycerides (mmol/L) \\
Waist circumference (cm) \\
No diabetes \\
Prediabetes \\
Diabetes mellitus \\
Treatment with OAD \\
Antihypertensive medication \\
Treatment with statins \\
Antidepressive medication \\
Antipsychotic medication \\
\hline
\end{tabular}

\begin{tabular}{c}
\hline Study A \\
\hline Anakinra $(n=73)$ \\
$54(11)$ \\
$36(49)$ \\
$37.4(5.5)$ \\
$72(99)$ \\
$1.3(2.0)$ \\
$13(18)$ \\
$68(93)$ \\
$136(17)$ \\
$84(10)$ \\
$78(11)$ \\
$3.59(3.34)$ \\
$51(69.9)$ \\
$4.56(1.12)$ \\
$1.35(0.71)$ \\
$2.45(0.97)$ \\
$9.46(8.73)$ \\
$7.4(3.2)$ \\
$2.16(1.45)$ \\
$122(13)$ \\
$10(13.7)$ \\
$16(21.9)$ \\
$47(64.4)$ \\
$36(49.3)$ \\
$53(72.6)$ \\
$35(47.9)$ \\
$11(15.1)$ \\
$1(1.4)$
\end{tabular}

\begin{tabular}{cc}
\hline \multicolumn{3}{c}{ Study B } \\
\hline Anakinra $(n=33)$ & Placebo $(n=33)$ \\
$55(13)$ & $53(14)$ \\
$33(100)$ & $33(100)$ \\
$36.8(4.1)$ & $36.6(4.0)$ \\
$33(100)$ & $33(100)$ \\
$4.2(5.9)$ & $4.6(5.5)$ \\
$11(33)$ & $5(15)$ \\
$33(100)$ & $32(97)$ \\
$140(16)$ & $136(15)$ \\
$88(12)$ & $86(9)$ \\
$70(10)$ & $69(12)$ \\
$3.04(2.07)$ & $4.01(3.30)$ \\
$25(75.8)$ & $22(66.7)$ \\
$5.08(1.72)$ & $4.64(1.45)$ \\
$1.17(0.26)$ & $1.50(1.25)$ \\
$4.02(6.89)$ & $2.80(1.00)$ \\
$8.59(4.96)$ & $8.52(5.06)$ \\
$6.1(1.0)$ & $5.9(0.6)$ \\
$2.28(1.71)$ & $2.11(1.37)$ \\
$124(10)$ & $127(19)$ \\
$14(42.4)$ & $18(54.5)$ \\
$10(30.3)$ & $8(24.2)$ \\
$9(27.3)$ & $7(21.2)$ \\
$9(27.3)$ & $6(18.2)$ \\
$16(48.5)$ & $14(42.4)$ \\
$11(33.3)$ & $7(21.2)$ \\
$1(3.0)$ & $1(3.0)$ \\
$0(0.0)$ & $0(0.0)$ \\
&
\end{tabular}

Variables are summarized as mean (s.D.) or counts (\%) if not otherwise specified.

\section{Effect of IL-1 receptor antagonism on copeptin levels}

Median copeptin levels at baseline were 7.0 (IQR 4.2-11.2) $\mathrm{pmol} / \mathrm{L}$ in the anakinra group and 7.2 (IQR 5.4-10.1) $\mathrm{pmol} / \mathrm{L}$ in the placebo group. After treatment initiation, copeptin levels did not change either in the anakinra or in the placebo group and remained stable throughout the treatment $(P=0.44$; Table 2, Fig. 2). Subgroup analyses were performed for (1) chronic low-grade inflammation, (2) diabetes status, (2) BMI below or above $35 \mathrm{~kg} / \mathrm{m}^{2}$, and (4) baseline copeptin levels according to a stratification of baseline copeptin values into tertile categories of baseline copeptin. The results are depicted in Fig. 3a, b, c, d. Treatment was no significant variable in any of the four subgroups. Only the interaction $P$ for chronic low-grade inflammation and treatment was below 0.05 , suggesting that anakinra has a different effect in patients with chronic low-grade inflammation at baseline compared to those without. However, data exploration revealed a high probability of a type I error due to randomly large differences in baseline copeptin levels between the two treatment groups in the subgroup without chronic low-grade inflammation. Furthermore, the effect size was very small (-0.7 pmol/L) and has no clinical relevance.

\section{Discussion}

In this analysis we present the following main findings: First, presence of (1) chronic low-grade inflammation, as shown with increased CRP levels, and (2) a BMI of >35 $\mathrm{kg} / \mathrm{m}^{2}$ was independently associated with higher copeptin levels in patients with metabolic syndrome. Second, treatment with the IL- 1 receptor antagonist anakinra did not lead to a reduction of copeptin levels in this patient population. There was also no relevant effect of IL-1 antagonism in any of the analyzed subgroups.

Numerous studies have described associations of high copeptin levels with the metabolic syndrome. Most of them found that this association did not persist after adjusting for the single components of the metabolic syndrome. However, according to several studies, copeptin levels remained independently associated with obesity $(6,7,21,22,34)$, insulin resistance $(7,10,22,34)$, and chronic low-grade inflammation $(6,8,22)$. These results are in accordance with our findings. 

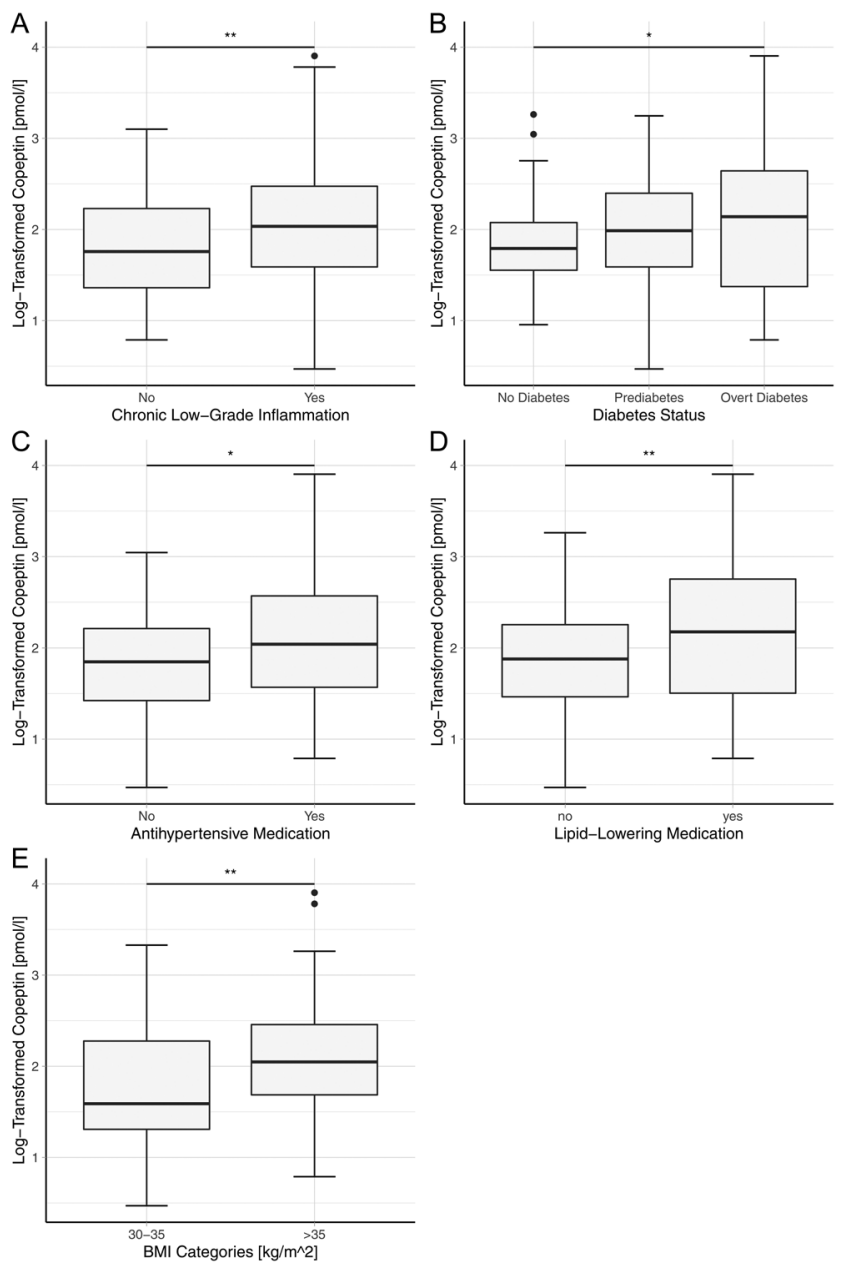

Figure 1

(A-E) Log-transformed copeptin values according to different subgroups. Log-transformed copeptin values measured at baseline are shown on the $y$-axis according to different subgroups. (A) Patients with and without chronic low-grade inflammation as defined by $C$-reactive protein values of $<$ or $\geq 2 \mathrm{mg} / \mathrm{L}$ at baseline. (B) Diabetic status was determined by medical history and HbA1c cut-offs, $\geq 6.5 \%$ as overt type 2 diabetes and $5.7-6.4 \%$ defined as prediabetes. (C and D) Patients with or without any antihypertensive $(C)$ and lipid-lowering $(D)$ medication at baseline. $(E)$ Subgroup according to BMI at baseline. $* P<0.05, * * P<0.01$.

Obesity, especially of the visceral type, is considered the driving factor for the development of insulin resistance and type 2 diabetes (35). Furthermore, visceral obesity evokes a chronic pro-inflammatory state (36). Chronic low-grade inflammation plays an important role in the destruction of the $\beta$-cells of the pancreas (37). Concordantly, it was shown that antagonism of the IL-1 pathway ameliorates glucose metabolism in patients with type 2 diabetes (25). There are experimental data showing stimulating effects of IL-1 on AVP secretion from the neurohypophysis $(27,28,29)$. Therefore, the increased copeptin levels observed in the metabolic syndrome might be induced by

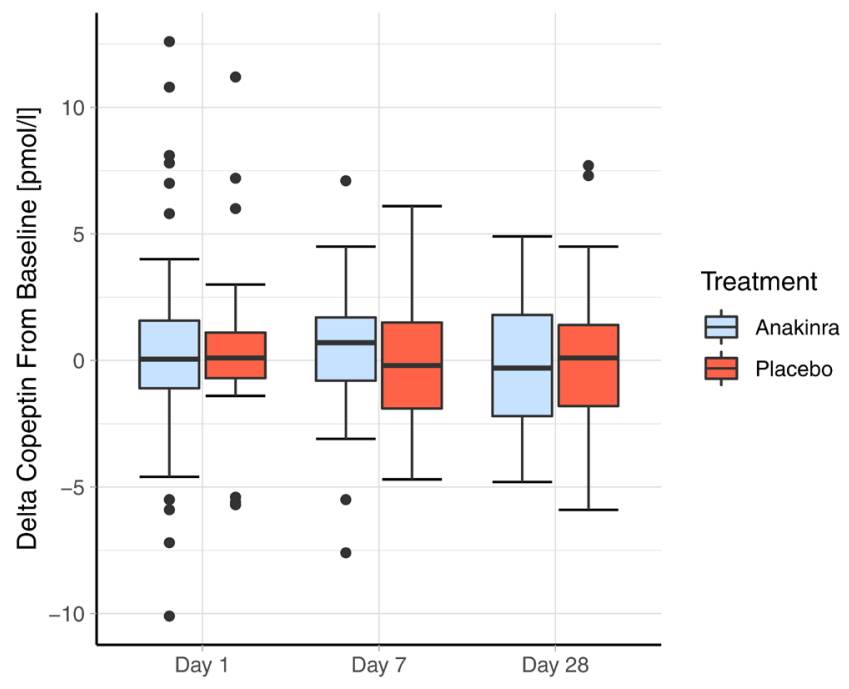

Figure 2

Change in Copeptin levels from baseline according to treatment group Copeptin values at day 1, 7 and 28 were subtracted from baseline and split according to the treatment group. The difference is depicted on the $y$-axis.

IL-1-driven chronic low-grade inflammation. In this case, blocking the IL-1 pathway would lead to a reduction of AVP/copeptin levels in patients with metabolic syndrome. To our knowledge, our study is the first to investigate the effects of IL-1 antagonism on the regulation of AVP/ copeptin levels in patients with metabolic syndrome. Interestingly, however, we did not observe any effects of the IL-1 antagonist anakinra on copeptin levels in our study patients. In the original studies, we showed that the IL-1 antagonist significantly down-regulated inflammation mirrored by CRP and interleukin- 6 levels as early as at day 1 of treatment $(31,32)$. Despite this clear anti-inflammatory action of anakinra, we observed no effect on copeptin levels. We, therefore, conclude that systemic IL-1 is not a major regulator of copeptin levels in the metabolic syndrome. Thus, the question, which factor leads to the upregulation of copeptin levels in patients with metabolic syndrome, remains open. Possibly, obesity might be the underlying factor for both, chronic low-grade inflammation and high copeptin levels. Visceral obesity represents a metabolic stress state leading not only to the secretion of proinflammatory cytokines (38), but also to a chronic activation of the sympathetic nervous system which is most obviously mirrored by elevation of blood pressure (39). Activation of the sympathetic nervous system is recognized as a non-osmotic stimulus for the secretion of AVP/copeptin (40). Therefore, it is possible that the chronic metabolic stress in obesity increases AVP/ copeptin levels, which is mediated by sympathetic nervous 
A Subgroup of Patients With

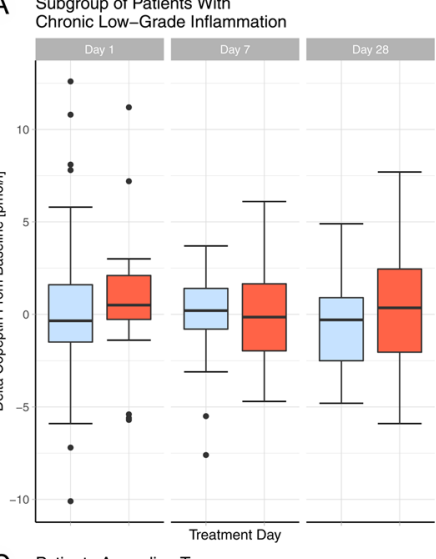

C Patients According To
BMI Categories

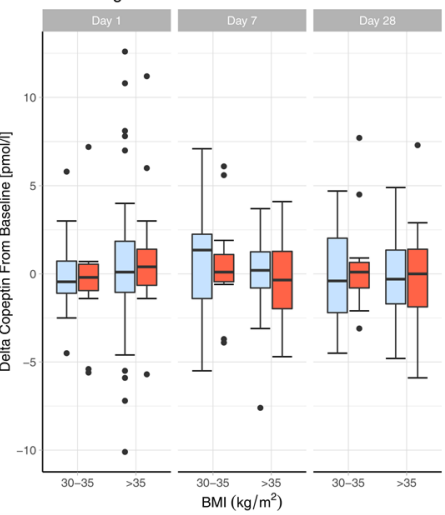

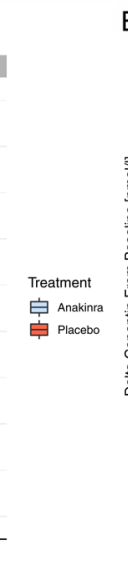

B Patients According To
Diabetic Status

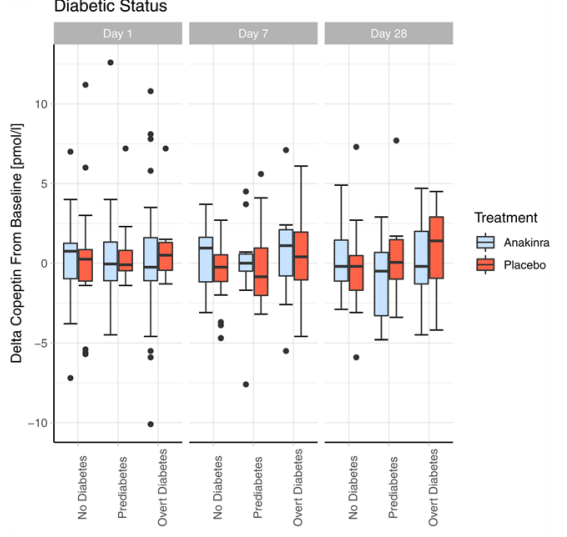

D Patients In The Highest Tertile of Baseline Copeptin Levels

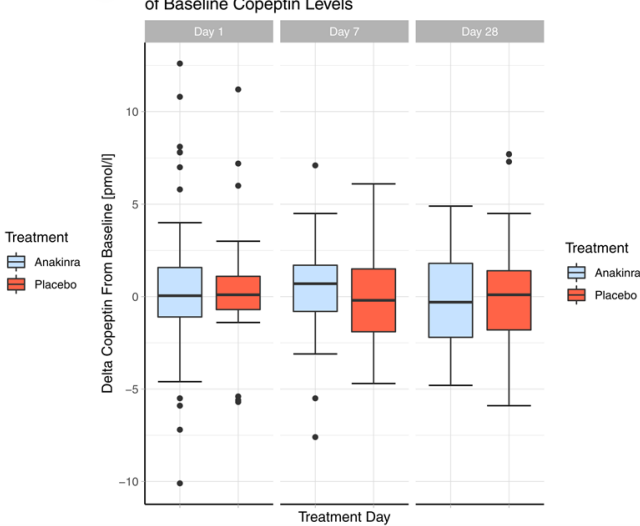

\section{Figure 3}

(A-D) Change in copeptin levels from baseline according to treatment group - subgroup analyses. Patients were divided into subgroups according to the presence of $(A)$ chronic low-grade inflammation, as defined by C-reactive protein values of $<$ or $\geq 2 \mathrm{mg} / \mathrm{L}$ at baseline, (B) diabetic status at baseline which was determined by medical history and $\mathrm{HbA} 1 \mathrm{c}$ cut-offs, $\geq 6.5 \%$ as overt type 2 diabetes and $5.7-6.4 \%$ defined as prediabetes, (C) according to BMI at baseline, (D) according to baseline copeptin levels in the highest tertile ( $\geq 9.4 \mathrm{pmol} / \mathrm{L}$ ). Copeptin values at day 1,7 and 28 were subtracted from baseline and split according to the treatment group. The difference in copeptin values according to the subgroup is depicted on the $y$-axis. activity. Interestingly, and in support of this hypothesis, Loncar et al. reported a reduction of copeptin levels after initiation of beta-blockade treatment in patients with heart failure and reduced ejection fraction (41).

As our study patients were already obese at study inclusion, we cannot conclude from our data whether obesity per se is the driving force for high copeptin levels. To investigate this hypothesis, data before and after weight gain through overfeeding or lack of physical exercise are required. However, surprisingly, we found only one abstract reporting study results on copeptin levels before and after weight loss so far. In this study by Aktimur et al., weight loss induced by bariatric surgery led to a significant decrease in copeptin levels, arguing for a causal role of obesity in high copeptin levels (42). Nevertheless, a bidirectional relationship between elevated AVP/copeptin levels and obesity needs to be considered. In this regard, Enhörning etal. showed in a longitudinal analysis that high copeptin levels at baseline predicted the development of abdominal obesity and type 2 diabetes after 15.8 years of follow-up (6). The authors suggested that AVP might play a causal role in the development of these two conditions by enhancing gluconeogenesis and glycogenolysis in the liver through vasopressin 1 a receptors $(43,44)$ and through antilipolytic effects (18). Furthermore, it might lead to hyperinsulinemia through activation of vasopressin $1 \mathrm{~b}$

Table 2 Effects of IL-1 receptor antagonism on copeptin levels.

\begin{tabular}{|c|c|c|c|c|}
\hline \multirow[b]{2}{*}{ Copeptin (pmol/L) } & \multicolumn{2}{|c|}{ Anakinra $(n=106, \boldsymbol{n}=73$ in Study A, $\boldsymbol{n}=33$ in Study B) } & \multicolumn{2}{|c|}{ Placebo $(n=33$, Study B) } \\
\hline & Absolute values & Change from baseline & Absolute values & Change from baseline \\
\hline Baseline & $7.0(4.2-11.2)$ & - & $7.2(5.4-10.1)$ & - \\
\hline Day 1 & 7.5 (3.9-11.5) & $0.1(-1.1-1.6)$ & $7.5(6.2-10.4)$ & $0.1(-0.7-1.1)$ \\
\hline Day 7 & $8.6(5.7-10.4)$ & $0.7(-0.8-1.7)$ & $7.2(5.6-10.0)$ & $-0.2(-1.9-1.5)$ \\
\hline Day 28 & $7.5(5.3-10.4)$ & $-0.3(-2.2-1.8)$ & $7.9(5.7-9.3)$ & $0.1(-1.8-1.4)$ \\
\hline
\end{tabular}

Variables are summarized as medians (IQR). For the rows 'Baseline' and 'Day 1', patients from both studies A and B were included. For 'Day 7' and 'Day 28', only data from study B were available.

https://ec.bioscientifica.com

https://doi.org/10.1530/EC-20-0197 (c) 2020 The authors Published by Bioscientifica Ltd

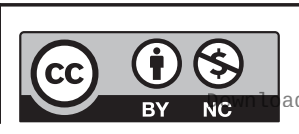

This work is licensed under a Creative Commons Attribution-NonCommercial 4.0 International License. ded from Bioscientifica.com at 04/26/2023 09:38:11AM via free access 
receptors in the pancreas (45). In summary, the available evidence suggests a bidirectional role of obesity for the secretion of $\mathrm{AVP} /$ copeptin. According to our study, however, chronic low-grade inflammation is probably not the driving force behind the elevation of AVP/copeptin levels and other mechanisms such as sympathetic nervous system activation must be investigated in future studies.

Strengths of our study are first that we used data from interventional studies, one being a placebo-controlled, double-blinded trial, which spares questions about association vs causality. Second, we investigated shortterm as well as longer-term effects of IL-1 antagonism on AVP/copeptin levels. Third, both studies had similar eligibility criteria and visit procedures. In both trials patients had to be fasting and refrain from drinking water before the morning blood samplings, rendering reliable copeptin measurements.

Limitations of our study include that this is a secondary analysis, which always bears the risk of insufficient power for this endpoint. Nevertheless, no tendency for a decrease in copeptin levels can be observed in our data. Alternatively, another cytokine (e.g. tumor necrosis factor $\alpha$ ) or cell nutrients (e.g. free fatty acids, glucose) may regulate AVP. Thus, anakinra alone might not be sufficiently potent to inhibit the drive of the other (unknown) factors on AVP/copeptin secretion.

In conclusion, the observed elevation of AVP/copeptin levels in patients with metabolic syndrome is not due to systemic chronic activation of the IL-1 system and other factors should be investigated to elucidate regulators of AVP/copeptin levels.

\section{Declaration of interest}

MCC received speaking honoraria from Thermo Fisher AG, the manufacturer of the Copeptin assay. The remaining authors have nothing to disclose.

\section{Funding}

MP and MCC are supported by grants awarded from the Swiss National Science Foundation (MCC: SNF-162608; MP: SNF-177578). MCC received speaking honoraria from Thermo Fisher AG, the manufacturer of the Copeptin assay. The remaining authors have nothing to disclose.

\section{Financial support}

MP and MCC are supported by grants awarded from the Swiss National Science Foundation (MCC: SNF-162608; MP: SNF-177578).

\section{Acknowledgements}

The authors thank all patients for their participation, the staff of the laboratory and the Department of Endocrinology, Diabetology \&
Metabolism of the University Hospital Basel and of the Kantonsspital Aarau. The authors extend a special thanks to the study nurses for their most helpful support during the study.

\section{References}

1 Eckel RH, Grundy SM \& Zimmet PZ. The metabolic syndrome. Lancet 2005365 1415-1428. (https://doi.org/10.1016/S01406736(05)66378-7)

2 Melander O. Vasopressin, from regulator to disease predictor for diabetes and cardiometabolic risk. Annals of Nutrition and Metabolism 201668 (Supplement 2) 24-28. (https://doi. org/10.1159/000446201)

3 Fenske WK, Schnyder I, Koch G, Walti C, Pfister M, Kopp P, Fassnacht M, Strauss K \& Christ-Crain M. Release and decay kinetics of copeptin vs AVP in response to osmotic alterations in healthy volunteers. Journal of Clinical Endocrinology \& Metabolism 2018103 505-513. (https://doi.org/10.1210/jc.2017-01891)

4 Enhörning S, Wang TJ, Nilsson PM, Almgren P, Hedblad B, Berglund G, Struck J, Morgenthaler NG, Bergmann A, Lindholm E, et al. Plasma copeptin and the risk of diabetes mellitus. Circulation 2010121 2102-2108. (https://doi.org/10.1161/ CIRCULATIONAHA.109.909663)

5 Abbasi A, Corpeleijn E, Meijer E, Postmus D, Gansevoort RT, Gans ROB, Struck J, Hillege HL, Stolk RP, Navis G, et al. Sex differences in the association between plasma copeptin and incident type 2 diabetes: the Prevention of Renal and Vascular Endstage Disease (PREVEND) study. Diabetologia 201255 1963-1970. (https:// doi.org/10.1007/s00125-012-2545-x)

6 Enhörning S, Bankir L, Bouby N, Struck J, Hedblad B, Persson M, Morgenthaler NG, Nilsson PM \& Melander O. Copeptin, a marker of vasopressin, in abdominal obesity, diabetes and microalbuminuria: the prospective Malmö Diet and Cancer Study cardiovascular cohort. International Journal of Obesity 201337 598-603. (https://doi. org/10.1038/ijo.2012.88)

7 Asferg CL, Andersen UB, Linneberg A, Goetze JP \& Jeppesen JL. Copeptin, a surrogate marker for arginine vasopressin secretion, is associated with higher glucose and insulin concentrations but not higher blood pressure in obese men. Diabetic Medicine 201431 728-732. (https://doi.org/10.1111/dme.12411)

8 Then C, Kowall B, Lechner A, Meisinger C, Heier M, Koenig W, Peters A, Rathmann W \& Seissler J. Plasma copeptin is associated with type 2 diabetes in men but not in women in the populationbased KORA F4 study. Acta Diabetologica 201552 103-112. (https:// doi.org/10.1007/s00592-014-0609-8)

9 Roussel R, Boustany R El, Bouby N, Potier L, Fumeron F, Mohammedi K, Balkau B, Tichet J, Bankir L, Marre M, et al. Plasma copeptin, AVP gene variants, and incidence of Type 2 diabetes in a cohort From the community. Journal of Clinical Endocrinology \& Metabolism 2016101 2432-2439. (https://doi.org/10.1210/jc.20161113)

10 Vintilă M, Gheorghiu ML, Caragheorgheopol A, Baculescu N, Lichiardopol C, Badiu C, Coculescu M, Grigorescu F \& Poiană C. Increased copeptin levels in metabolic syndrome from a Romanian population. Journal of Medicine and Life 20169 353-357.

11 Riphagen IJ, Boertien WE, Alkhalaf A, Kleefstra N, Gansevoort RT, Groenier KH, van Hateren KJJ, Struck J, Navis G, Bilo HJG, et al. Copeptin, a surrogate marker for arginine vasopressin, is associated with cardiovascular and all-cause mortality in patients with type 2 diabetes (ZODIAC-31). Diabetes Care 201336 3201-3207. (https:// doi.org/10.2337/dc12-2165)

12 Zellweger C, Wildi K, Twerenbold R, Reichlin T, Naduvilekoot A, Neuhaus JD, Balmelli C, Gabutti M, Afify AAl, Ballarino P, et al. Use of copeptin and high-sensitive cardiac troponin $\mathrm{T}$ for diagnosis and prognosis in patients with diabetes mellitus and suspected acute 
myocardial infarction. International Journal of Cardiology 2015190 190-197. (https://doi.org/10.1016/j.ijcard.2015.04.134)

13 Enhörning S, Hedblad B, Nilsson PM, Engström G \& Melander O. Copeptin is an independent predictor of diabetic heart disease and death. American Heart Journal 2015169 549-556.e1. (https://doi. org/10.1016/J.AHJ.2014.11.020)

14 Gallo-Payet N \& Guillon G. Regulation of adrenocortical function by vasopressin. Hormone and Metabolic Research 199830 360-367. (https://doi.org/10.1055/s-2007-978899)

15 Scott LV \& Dinan TG. Vasopressin and the regulation of hypothalamic-pituitary-adrenal axis function: implications for the pathophysiology of depression. Life Sciences 199862 1985-1998. (https://doi.org/10.1016/s0024-3205(98)00027-7)

16 Grazzini E, Breton C, Derick S, Andres M, Raufaste D, Rickwaert F, Boccara G, Colson P, Guérineau NC, Serradeil-Le Gal C, et al. Vasopressin receptors in human adrenal medulla and pheochromocytoma. Journal of Clinical Endocrinology \& Metabolism 199984 2195-2203. (https://doi.org/10.1210/jcem.84.6.5775)

17 Hems DA \& Whitton PD. Stimulation by vasopressin of glycogen breakdown and gluconeogenesis in the perfused rat liver. Biochemical Journal 1973136 705-709. (https://doi.org/10.1042/bj1360705)

18 Hiroyama M, Aoyagi T, Fujiwara Y, Birumachi J, Shigematsu Y, Kiwaki K, Tasaki R, Endo F \& Tanoue A. Hypermetabolism of fat in V1a vasopressin receptor knockout mice. Molecular Endocrinology 200721 247-258. (https://doi.org/10.1210/me.2006-0069)

19 Filep J \& Rosenkranz B. Mechanism of vasopressin-induced platelet aggregation. Thrombosis Research 198745 7-15. (https://doi. org/10.1016/0049-3848(87)90252-0)

20 Maturi MF, Martin SE, Markle D, Maxwell M, Burruss CR, Speir E, Greene R, Ro YM, Vitale D \& Green MV. Coronary vasoconstriction induced by vasopressin. Production of myocardial ischemia in dogs by constriction of nondiseased small vessels. Circulation $1991 \mathbf{8 3}$ 2111-2121. (https://doi.org/10.1161/01.cir.83.6.2111)

21 Enhörning S, Struck J, Wirfält E, Hedblad B, Morgenthaler NG $\&$ Melander O. Plasma copeptin, a unifying factor behind the metabolic syndrome. Journal of Clinical Endocrinology \& Metabolism 201196 E1065-E1072. (https://doi.org/10.1210/jc.2010-2981)

22 Wannamethee SG, Welsh P, Papacosta O, Lennon L, Whincup PH \& Sattar N. Copeptin, insulin resistance, and risk of incident diabetes in older men. Journal of Clinical Endocrinology \& Metabolism 2015100 3332-3339. (https://doi.org/10.1210/JC.2015-2362)

23 Barchetta I, Enhörning S, Cimini FA, Capoccia D, Chiappetta C, Cristofano C Di, Silecchia G, Leonetti F, Melander O \& Cavallo MG. Elevated plasma copeptin levels identify the presence and severity of non-alcoholic fatty liver disease in obesity. BMC Medicine 20191785. (https://doi.org/10.1186/s12916-019-1319-4)

24 Ridker PM, Howard CP, Walter V, Everett B, Libby P, Hensen J, Thuren T \& CANTOS Pilot Investigative Group. Effects of interleukin- $1 \beta$ inhibition with canakinumab on hemoglobin A1c, lipids, C-reactive protein, interleukin-6, and fibrinogen a phase IIb randomized, placebo-controlled trial. Circulation 2012126 2739-2748. (https://doi.org/10.1161/ CIRCULATIONAHA.112.122556)

25 Larsen CM, Faulenbach M, Vaag A, Vølund A, Ehses JA, Seifert B, Mandrup-Poulsen T \& Donath MY. Interleukin-1-receptor antagonist in type 2 diabetes mellitus. New England Journal of Medicine 2007356 1517-1526. (https://doi.org/10.1056/NEJMoa065213)

26 Ridker PM, Everett BM, Thuren T, MacFadyen JG, Chang WH, Ballantyne C, Fonseca F, Nicolau J, Koenig W, Anker SD, et al. Antiinflammatory therapy with canakinumab for atherosclerotic disease. New England Journal of Medicine 2017377 1119-1131. (https://doi.org/10.1056/NEJMoa1707914)

27 Nakatsuru K, Ohgo S, Oki Y \& Matsukura S. Interleukin-1 (IL-1) stimulates arginine vasopressin (AVP) release from superfused rat hypothalamo-neurohypophyseal complexes independently of cholinergic mechanism. Brain Research 1991554 38-45. (https://doi. org/10.1016/0006-8993(91)90169-v)

28 Watanobe H \& Takebe K. Intrahypothalamic perfusion with interleukin-1-beta stimulates the local release of corticotropinreleasing hormone and arginine vasopressin and the plasma adrenocorticotropin in freely moving rats: a comparative perfusion of the paraventricular nucleus and the median eminence. Neuroendocrinology 199357 593-599. (https://doi. org/10.1159/000126412)

29 Raber J, Pich EM, Koob GF \& Bloom FE. IL-1 beta potentiates the acetylcholine-induced release of vasopressin from the hypothalamus in vitro, but not from the amygdala. Neuroendocrinology 199459 208-217. (https://doi.org/10.1159/000126661)

30 Wahab F, Tazinafo LF, Cárnio EC, Aguila FA, Batalhão ME \& Rocha MJ. Interleukin-1 receptor antagonist decreases cerebrospinal fluid nitric oxide levels and increases vasopressin secretion in the late phase of sepsis in rats. Endocrine 201549 215-221. (https://doi. org/10.1007/s12020-014-0452-2)

31 Ebrahimi F, Urwyler SA, Straumann S, Doerpfeld S, Bernasconi L, Neyer P, Schuetz P, Mueller B, Donath MY \& Christ-Crain M. IL-1 antagonism in men with metabolic syndrome and low testosterone: a randomized clinical trial. Journal of Clinical Endocrinology and Metabolism 2018103 3466-3476. (https://doi.org/10.1210/jc.201800739)

32 Urwyler SA, Schuetz P, Ebrahimi F, Donath MY \& Christ-Crain M. Interleukin-1 antagonism decreases cortisol levels in obese individuals. Journal of Clinical Endocrinology \& Metabolism 2017102 1712-1718. (https://doi.org/10.1210/jc.2016-3931)

33 Friedewald WT, Levy RI \& Fredrickson DS. Estimation of the concentration of low-density lipoprotein cholesterol in plasma, Without use of the preparative ultracentrifuge. Clinical Chemistry 197218 499-502. (https://doi.org/10.1093/ clinchem/18.6.499)

34 Saleem U, Khaleghi M, Morgenthaler NG, Bergmann A, Struck J, Mosley TH \& Kullo IJ. Plasma carboxy-terminal provasopressin (copeptin): a novel marker of insulin resistance and metabolic syndrome. Journal of Clinical Endocrinology \& Metabolism 200994 2558-2564. (https://doi.org/10.1210/jc.2008-2278)

35 Kahn BB \& Flier JS. Obesity and insulin resistance. Journal of Clinical Investigation 2000106 473-481. (https://doi.org/10.1172/ JCI10842)

36 Monteiro R \& Azevedo I. Chronic inflammation in obesity and the metabolic syndrome. Mediators of Inflammation 20102010289645. (https://doi.org/10.1155/2010/289645)

37 Aharon-Hananel G, Jörns A, Lenzen S, Raz I \& Weksler-Zangen S. Antidiabetic effect of interleukin- $1 \beta$ antibody therapy through $\beta$-cell protection in the Cohen diabetes - sensitive rat. Diabetes 201564 1780-1785. (https://doi.org/10.2337/db14-1018)

38 Donath MY. Inflammation as a sensor of metabolic stress in obesity and type 2 diabetes. Endocrinology 2011152 4005-4006. (https://doi. org/10.1210/en.2011-1691)

39 Davy KP \& Orr JS. Sympathetic nervous system behavior in human obesity. Neuroscience and Biobehavioral Reviews 200933 116-124. (https://doi.org/10.1016/j.neubiorev.2008.05.024)

40 Schrier RW \& Goldberg JP. The physiology of vasopressin release and the pathogenesis of impaired water excretion in adrenal, thyroid, and edematous disorders. Yale Journal of Biology and Medicine 198053 525-541.

41 Loncar G, von Haehling S, Tahirovic E, Inkrot S, Mende M, Sekularac N, Lainscak M, Apostolovic S, Putnikovic B, Edelmann F, et al. Effect of beta blockade on natriuretic peptides and copeptin in elderly patients with heart failure and preserved or reduced ejection fraction: results from the CIBIS-ELD trial. Clinical Biochemistry 201245 117-122. (https://doi.org/10.1016/j. clinbiochem.2011.11.010) https://ec.bioscientifica.com https://doi.org/10.1530/EC-20-0197 (c) 2020 The authors Published by Bioscientifica Ltd

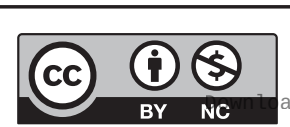

This work is licensed under a Creative Commons Attribution-NonCommercial 4.0 International License. ded from Bioscientifica.com at 04/26/2023 09:38:11AM 
42 Aktimur R, Mete T, Cetinkunar S, Yaman M, Beton O, Avci E, Erdem $\mathrm{H} \&$ Yildirim K. Copeptin, a marker of vasopressin, decreases significantly in early state after bariatric surgery. Endocrine Abstracts 201641 EP806. (https://doi.org/10.1530/endoabs.41.EP806)

43 Whitton PD, Rodrigues LM \& Hems DA. Stimulation by vasopressin, angiotensin and oxytocin of gluconeogenesis in hepatocyte suspensions. Biochemical Journal 1978176 893-898. (https://doi. org/10.1042/bj1760893)
44 Keppens S \& De WH. The nature of the hepatic receptors involved in vasopressin-induced glycogenolysis. BBA - Series General Subjects 1979588 63-69. (https://doi.org/10.1016/03044165(79)90371-4)

45 Abu-Basha EA, Yibchok-Anun S \& Hsu WH. Glucose dependency of arginine vasopressin-induced insulin and glucagon release from the perfused rat pancreas. Metabolism: Clinical and Experimental 200251 1184-1190. (doi:10.1053/meta.2002.34052)

Received in final form 10 June 2020

Accepted 1 July 2020

Accepted Manuscript published online 2 July 2020
This work is licensed under a Creative Commons Attribution-NonCommercial 4.0 International License. 